\title{
Evaluation of FGF-19 and $\beta$-klotho as biomarkers in patients with intrahepatic cholestasis of pregnancy
}

Ahter Tanay Tayyar ${ }^{1}$, Ahmet Tayyar ${ }^{2}$, Sukran Kozali ${ }^{1}$, Resul Karakus ${ }^{1}$, Nadiye Koroglu², Ilkbal Temel Yuksel², Gonca Yetkin Yildirim², Ismail Dag³, Mustafa Eroglu ${ }^{1}$

\begin{abstract}
${ }^{1}$ Department of Obstetrics and Gynecology, Health Sciences University Zeynep Kamil Maternity and Children's Research Hospital, Istanbul, Turkey

${ }^{2}$ Department of Obstetrics and Gynecology, Health Sciences University Kanuni Sultan Suleyman Training and Research Hospital, Istanbul, Turkey

${ }^{3}$ Department of Clinical Biochemistry, Eyup State Hospital, Istanbul, Turkey
\end{abstract}

Submitted: 18 November 2017

Accepted: 19 December 2017

Arch Med Sci 2019; 15, 1: 113-119

DOI: https://doi.org/10.5114/aoms.2017.72424

Copyright @ 2018 Termedia \& Banach

\section{Abstract}

Introduction: Fibroblast growth factor-19 (FGF-19) and its co-receptor, beta-klotho, regulate bile acid synthesis in the liver as an enterohepatic feedback mechanism. In this study, our aim was to investigate the circulating FGF-19 and $\beta$-klotho levels in intrahepatic cholestasis of pregnancy (ICP) cases.

Material and methods: A cross-sectional study including 40 women whose pregnancies were complicated with ICP were recruited for the study group. Forty randomly selected healthy pregnant women comprised the control group. The patient characteristics, including maternal age, gravidity, parity, gestational age at the time of diagnosis, body mass index (BMI), and obstetric history, were recorded. The serum FGF-19 and $\beta$-klotho concentrations were measured using an enzyme-linked immunosorbent assay.

Results: Maternal age, gravidity, parity, body mass index at assessment, and gestational age at blood sampling were similar between the two groups $(p>0.05)$. Moreover, there were no significant differences in the FGF-19 and $\beta$-klotho concentrations between the two groups ( $p=0.341$ and $p=0.086$, respectively). A positive correlation was detected between the $\beta$-klotho and FGF-19 levels, as well as between the FGF-19 level and BMI $(r=0.368$, $p=0.020$ and $r=0.389, p=0.013$, respectively).

Conclusions: The serum FGF-19 and $\beta$-klotho concentrations did not differ between the pregnancies with ICP and the healthy controls. However, in some cases, abnormalities in the FGF-19, $\beta$-klotho, and FGFR4 signaling system may play roles in the pathogenesis of ICP.

Key words: fibroblast growth factor-19, $\beta$-klotho, intrahepatic cholestasis of pregnancy, pathogenesis, CYP7A1.

\section{Introduction}

Intrahepatic cholestasis of pregnancy (ICP) is the most frequently occurring liver disease during pregnancy, with an incidence ranging between $0.1 \%$ and $15.6 \%$ [1]. The onset of this pregnancy-specific disease occurs during the late phases of the second and third trimesters, and it is characterized by itching (especially on the skin of the palms and soles), impaired liver function tests, and increased serum bile acid levels. However, its clinical and laboratory symptoms regress rapidly after birth [2].

\author{
Corresponding author: \\ Ahter Tanay Tayyar MD \\ Department of Obstetrics \\ and Gynecology \\ Zeynep Kamil Maternity \\ and Children's \\ Research Hospital \\ Istanbul, Turkey \\ Phone: +90 2163910680 \\ Fax: +90 2163910690 \\ E-mail: drahtertayyar@yahoo. \\ com
}


Intrahepatic cholestasis of pregnancy carries some importance in that it constitutes a risk for serious obstetric complications, such as a spontaneous or iatrogenic preterm delivery, fetal distress, sudden intrauterine fetal death, preeclampsia, gestational diabetes mellitus, and severe subsequent hepatobiliary disease in the affected women [3-6]. Abnormal biliary transport and a decrease in the bile acid excretion play roles in its etiology, whereas its pathophysiology has yet not been elucidated completely [7-9].

Bile acids are synthesized from cholesterol in the liver, which is important for the absorption of lipids and lipid-soluble materials. The appropriate control of bile acid synthesis plays a crucial role in the prevention of the toxic effects of bile acids and cholestasis [10]. Moreover, $7 \alpha$-hydroxylase (CYP7A1), which plays a role in the synthesis of bile acids, constitutes the rate-limiting step of the synthesis. It is strictly regulated by nuclear receptor pathways, such as the farnesoid $X$ receptor (FXR or NR1H4), and the negative feedback of the enterohepatic circulation $[10,11]$.

Fibroblast growth factor-19 (FGF-19) is an endocrine hormone essentially expressed by the intestinal epithelial cells of the ileum, and it is activated by increases in the bile acids [11-13]. As a co-receptor of FGF-19, the transmembrane protein $\beta$-klotho is another regulator of bile acid homeostasis, and it is expressed in the liver, pancreas, and adipose tissue [12, 14, 15]. Following its activation in the bowels, FGF-19 enters the enterohepatic circulation, where it is bound to fibroblast growth factor receptor-4 (FGFR4) found in the hepatocytes through a process mediated by $\beta$-klotho, and decreases the transcription of CYP7A1 [10, 13, $16,17]$. Via negative feedback, it inhibits bile acid synthesis, decreases the bile acid levels, and prevents the liver damage that might occur due to the toxic effects of the bile acids [10,11].

In light of these findings, the objective of this study was to investigate the serum levels of the FGF-19 protein (which regulates bile acid synthesis) and $\beta$-klotho in patients with ICP, and to contribute to the elucidation of ICP pathophysiology.

\section{Material and methods}

\section{Study design}

This cross-sectional study was conducted according to the principles of the Helsinki Declaration and it was approved by the local institutional review board (Decision No: 114/2017). Written informed consent was obtained from all of the subjects.

A total of 80 pregnant women admitted to the Health Sciences University Zeynep Kamil Research and Training Hospital and Kanuni Sultan Suleyman
Research and Training Hospital in Turkey constituted the study group. This study was conducted between March and September of 2017. A total of 40 consecutive pregnant women with ICP and 40 healthy women with uncomplicated pregnancies (control group) in their third trimesters were included in this study, after matching for age and gestational age. None of the patients were in labor at the time of inclusion.

Those patients whose pruritus could not be otherwise explained and those with increased fasting bile acid $(10 \mathrm{mmol} / \mathrm{l})$ and hepatic aminotransferase levels were considered to have ICP. We excluded women with any alternative causes of hepatic impairment, such as a history of gallstones or gallbladder disease, hepatotoxic drug consumption, hepatitis, and active infections or chronic inflammatory conditions, including inflammatory bowel disease, rheumatic disease, and vasculitis. Those patients taking any medications (including progesterone) except iron supplements at the time of hospital admission were excluded from the study. We also excluded those with multiple pregnancies, chromosomal abnormalities, fetal anomalies, maternal infections, itching with elevated liver enzymes and normal bile acids, pruritus with skin lesions, and pregnancies complicated by comorbidities, such as thyroid disease, hypertension, and diabetes mellitus, from the study groups.

\section{Outcome parameters}

We determined the gestational age by measuring the fetal crown-rump length at 11-13 weeks of gestation. The patient characteristics, including the maternal age, gravidity, parity, gestational age at the time of diagnosis, body mass index (BMI), and obstetric history, were recorded.

The fasting blood samples for the FGF-19 and $\beta$-klotho analyses were collected before starting treatment with ursodeoxycholic acid. A 5-cc venous blood sample was drawn into a biochemistry tube and centrifuged for $10 \mathrm{~min}$ at 4,000 g. The supernatants of the serum samples were then transferred into clean $1.5 \mathrm{ml}$ Eppendorf tubes and stored at $-80^{\circ} \mathrm{C}$ for enzyme-linked immunosorbent assay (ELISA) analysis. The laboratory results of the biochemical parameters and bile acid levels for each participant were recorded prospectively. The participants were followed up until delivery, and none of the patients in the control group developed ICP. Finally, a retrospective analysis of the biomarkers (FGF-19 and $\beta$-klotho) from the stored serum samples was performed by investigators who were blinded to all of the clinical data.

The serum $\beta$-klotho concentrations were measured using a sandwich enzyme-linked immunosorbent assay kit (Human Klotho beta PicoKine ELISA Kit, catalog no. EK1492; Boster Biological 
Technology, Pleasanton, CA, USA). The assay sensitivity was $<10 \mathrm{pg} / \mathrm{ml}$, and the inter-assay and intra-assay calculation values were $<7.4 \%$ and $<6.8 \%$, respectively. The serum FGF-19 concentrations were also measured using a sandwich enzyme-linked immunosorbent assay kit (Human FGF19 PicoKine ELISA Kit, catalog no. EK1160; Boster Biological Technology, Pleasanton, CA, USA). The assay sensitivity was $<10 \mathrm{pg} / \mathrm{ml}$, and the inter-assay and intra-assay calculation values were $<7.2 \%$ and $<6.5 \%$, respectively.

\section{Statistical analysis}

The data were analyzed using the MedCalc statistical software version 17.8 (MedCalc Software, Ostend, Belgium; http://www.medcalc.org, 2017) for the statistical analyses. The normal distribution of the quantitative data was checked using the Kolmogorov-Smirnov test. Parametric tests (independent-samples $t$-test) were applied to the data with a normal distribution, and non-parametric tests (Mann-Whiney U-test and Kruskal-Wallis test) were applied when the data were not normally distributed. If the values were significantly different in the Kruskal-Wallis test, a Conover post hoc test was performed. The correlations were assessed using Spearman's correlation coefficient, along with the related $p$-values. The data were expressed as the mean \pm SD or median (interquartile range), as appropriate. Statistical significance was assumed for $p<0.05$.

\section{Results}

Forty patients with ICP and 40 healthy pregnant women were included in this study, and the characteristic features and laboratory findings of the study groups are summarized in Table I. The maternal ages, gravidities, parities, BMls at assessment, and gestational ages at blood sampling were similar between the two groups $(p>0.05)$; however, the gestational weeks and birth weights were significantly higher in the control group $(p<0.05)$. The liver function tests, $\gamma$-glutamyl transpeptidase (GGT) levels, direct bilirubin, and fasting total bile acid (TBA) levels were significantly higher in the ICP group than in the control group $(p<0.001, p=0.009, p<0.0001$, and $p<0.0001$, respectively).

The serum $\beta$-klotho level was lower in the ICP group than in the control group, without any significant intergroup difference $(p=0.086)$. Moreover, the serum FGF-19 levels were not significantly different between the groups ( $p=0.341$ ) (Table I, Figure 1). The correlation between the parameters

Table I. Characteristics and laboratory parameters of intrahepatic cholestasis of pregnancy and control patients

\begin{tabular}{|c|c|c|c|}
\hline Parameter & $\begin{array}{l}\text { Controls } \\
(n=40)\end{array}$ & $\begin{array}{l}\text { Cholestasis } \\
\qquad(n=40)\end{array}$ & $P$-values \\
\hline Age [years] ${ }^{*}$ & $27.35 \pm 6.16$ & $27.28 \pm 4.84$ & 0.952 \\
\hline Gravida, $n(\%)$ & $2(3)$ & $2(2)$ & 0.179 \\
\hline Parity, $n(\%)$ & $1(2)$ & $1(2)$ & 0.391 \\
\hline Parous, $n(\%)^{\dagger}$ & $23(57.5)$ & $22(55)$ & 0.882 \\
\hline BMI $\left[\mathrm{kg} / \mathrm{m}^{2}\right]^{*}$ & $27.84 \pm 3.60$ & $28.12 \pm 3.53$ & 0.719 \\
\hline 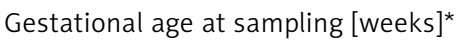 & $34.30 \pm 2.39$ & $34.54 \pm 2.73$ & 0.680 \\
\hline Gestational age at delivery [weeks] ${ }^{*}$ & $38.89 \pm 1.38$ & $36.49 \pm 1.58$ & $<0.0001$ \\
\hline Birth weight $[g]^{*}$ & $3305.9 \pm 374.5$ & $2927.8 \pm 509.7$ & $<0.0001$ \\
\hline $\mathrm{H} / \mathrm{O} \mathrm{ICP}, n(\%)^{\dagger}$ & 0 & $8(36.4)$ & 0.005 \\
\hline ALT [IU/I] & $14.5(12)$ & $81(138)$ & $<0.0001$ \\
\hline AST [IU/I] & $18(7)$ & $51.5(78)$ & $<0.0001$ \\
\hline GGT $[I U / I]^{*}$ & $16.1 \pm 4.9$ & $21.75 \pm 12.3$ & 0.009 \\
\hline Direct bilirubin $[\mathrm{mg} / \mathrm{dl}]^{*}$ & $0.05 \pm 0.02$ & $0.31 \pm 0.17$ & $<0.0001$ \\
\hline TBA $[\mu \mathrm{mol} / \mathrm{l}]$ (fasting) & $4.9(2.1)$ & $19.8(18.3)$ & $<0.0001$ \\
\hline$\beta$-klotho [pg/ml] & $528.74(826.09)$ & $439.51(398.79)$ & 0.086 \\
\hline FGF-19 [pg/ml] & $52.53(45.35)$ & 52.17 (19.39) & 0.341 \\
\hline
\end{tabular}

$B M I$ - body mass index, H/O ICP - previous history of intrahepatic cholestasis of pregnancy among the multipara, ALT - alanine aminotransferase, AST - aspartate aminotransferase, GGT - $\gamma$-glutamyl transferase, ICP - intrahepatic cholestasis of pregnancy, $T B A$ - total bile acids. The data are expressed as the median (interquartile range), unless otherwise indicated. ${ }^{*}$ Data expressed as mean $\pm S D .{ }^{\dagger}$ Analysis via Fisher's exact test. 
A

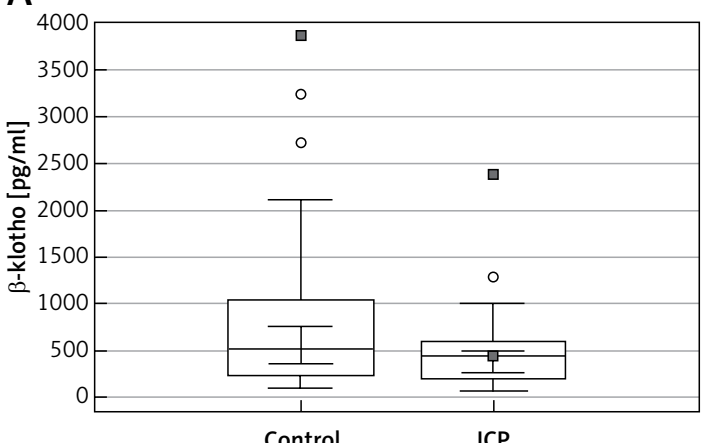

B

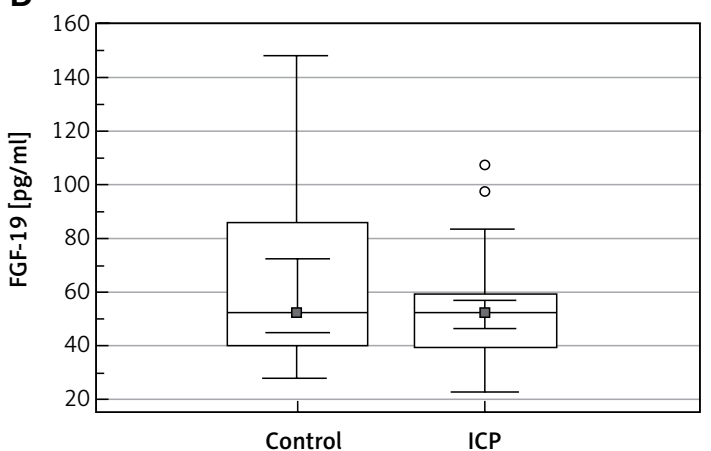

Figure 1. Boxplot analysis of (A) $\beta$-klotho and (B) FGF-19 levels in the patients with intrahepatic cholestasis of pregnancy and the control patients

Table II. Correlation analyses between maternal serum $\beta$-klotho, FGF-19, and the other parameters in the ICP group

\begin{tabular}{|c|c|c|c|c|c|c|c|c|c|c|c|}
\hline \multicolumn{2}{|c|}{ Parameter } & $\begin{array}{l}\text { FGF-19 } \\
{[\mathrm{pg} / \mathrm{ml}]}\end{array}$ & $\begin{array}{c}\beta \text {-klotho } \\
\text { [pg/ml] }\end{array}$ & $\begin{array}{c}\text { TBA } \\
{[\mu \mathrm{mol} / \mathrm{l}]}\end{array}$ & $\begin{array}{c}\text { Age } \\
\text { [year] }\end{array}$ & $\begin{array}{c}\text { BMI } \\
{\left[\mathrm{kg} / \mathrm{m}^{2}\right]}\end{array}$ & $\begin{array}{c}\text { GA } \\
\text { sampling } \\
\text { [weeks] }\end{array}$ & $\begin{array}{c}\text { ALT } \\
{[I U / I]}\end{array}$ & $\begin{array}{c}\text { AST } \\
{[\mathrm{IU} / \mathrm{I}]}\end{array}$ & $\begin{array}{c}\text { GGT } \\
{[I U / I]}\end{array}$ & $\begin{array}{c}\text { Direct } \\
\text { bilirubin } \\
\text { [mg/dl] }\end{array}$ \\
\hline \multirow{2}{*}{$\begin{array}{l}\beta \text {-klotho } \\
{[\mathrm{pg} / \mathrm{ml}]}\end{array}$} & $r_{s}$ & $0.368^{*}$ & & 0.70 & 0.235 & 0.096 & 0.042 & -0.097 & 0.013 & 0.306 & -0.174 \\
\hline & $p$ & 0.020 & & 0.666 & 0.144 & 0.556 & 0.795 & 0.551 & 0.938 & 0.055 & 0.283 \\
\hline \multirow{2}{*}{$\begin{array}{l}\text { FGF-19 } \\
{[\mathrm{pg} / \mathrm{ml}]}\end{array}$} & $r_{s}$ & & $0.368^{*}$ & -0.009 & 0.160 & $0.389^{*}$ & -0.062 & 0.114 & 0.094 & 0.080 & 0.096 \\
\hline & $p$ & & 0.020 & 0.954 & 0.323 & 0.013 & 0.702 & 0.483 & 0.562 & 0.622 & 0.554 \\
\hline
\end{tabular}

${ }^{*}$ Correlation is significant at the 0.05 level (2-tailed). BMI - body mass index, ALT - alanine aminotransferase, AST - aspartate aminotransferase, GGT - $\gamma$-glutamyl transferase, ICP - intrahepatic cholestasis of pregnancy, TBA - total bile acids, GA - gestational age.

of the ICP group and the $\beta$-klotho and FGF-19 levels is shown in Table II. A positive correlation was detected between the $\beta$-klotho and FGF-19 levels, as well as between the FGF-19 level and BMI $(r=$ $0.368, p=0.020$ and $r=0.389, p=0.013$, respectively).

Table III shows the subgroup analyses of parous women, in which the ICP patients without previous histories of ICP $(n=14)$, those who developed ICP previously $(n=8)$, and the control group $(n=23)$ were compared. A significant intergroup

Table III. Levels of tested biomarkers in patients with intrahepatic cholestasis of pregnancy with a history of intrahepatic cholestasis of pregnancy and without a history of intrahepatic cholestasis of pregnancy and the control group

\begin{tabular}{|lcc|}
\hline Variable & $\begin{array}{c}\beta \text {-klotho } \\
{[\mathrm{pg} / \mathrm{ml}]}\end{array}$ & $\begin{array}{c}\text { FGF-19 } \\
{[\mathrm{pg} / \mathrm{ml}]}\end{array}$ \\
\hline $\begin{array}{l}\text { ICP without H/O ICP } \\
(n=14)\end{array}$ & $468.84(463.24)$ & $54.58(21.94)$ \\
\hline $\begin{array}{l}\text { ICP with H/O ICP } \\
(n=8)\end{array}$ & $221.27(211.68)$ & $41.84(25.19)$ \\
\hline Controls $(n=23)$ & $520.56(802.70)$ & $48.20(38.75)$ \\
\hline$P$-values & 0.017 & 0.461 \\
\hline
\end{tabular}

ICP - intrahepatic cholestasis of pregnancy, H/O ICP - previous history of intrahepatic cholestasis of pregnancy among the multipara. Values are given as the median (interquartile range). Group medians were compared using the Kruskal-Wallis test, followed by the Conover post hoc analysis for the two-group pairwise comparisons. difference was detected for $\beta$-klotho levels ( $p=$ 0.017), whereas there was no significant difference in the FGF-19 concentrations between the groups ( $p=0.461$ ) (Figure 2 ).

\section{Discussion}

To the best of our knowledge, this was the first study to evaluate the roles of FGF-19 and

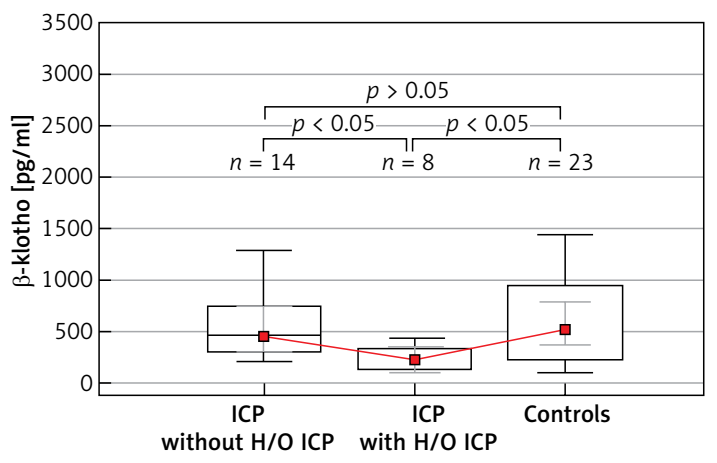

Figure 2. Boxplot analysis of serum $\beta$-klotho levels in patients with intrahepatic cholestasis of pregnancy with a history of intrahepatic cholestasis of pregnancy and without a history of intrahepatic cholestasis of pregnancy and the control group. The $p$-values presented in the figure were computed by a pairwise comparison of the subgroups using the Conover post hoc test following the Kruskal-Wallis test

ICP - intrahepatic cholestasis of pregnancy, H/O ICP previous history of intrahepatic cholestasis of pregnancy among the multipara. 
$\beta$-klotho in women with ICP. Our hypothesis was that the serum FGF-19 and $\beta$-klotho levels in ICP may demonstrate alterations when compared with those of healthy pregnant women; consequently, the regulation of the CYP7A1 enzyme, which is the rate-limiting step, is altered, leading to an increase in the bile acid levels. However, in our study, we did not detect a significant difference between the cholestasis and control groups with regard to the FGF-19 and $\beta$-klotho levels ( $p=0.341$ and $p=0.086$, respectively). Although it was not statistically significant, we detected a lower $\beta$-klotho level relative to the control group (439.51 (398.79) pg/ml vs. 528.74 (826.09) pg/ml). In our subgroup analysis, the $\beta$-klotho level was significantly lower in patients with previous histories of ICP $(p=0.017)$.

Intrahepatic cholestasis of pregnancy is a pregnancy-specific liver disease characterized by itching and impaired liver function tests $[18,19]$. Its risk factors may include advanced maternal age (> 35 years old), multiparity, family history, history of gestational cholestasis, and oral contraceptive use. The recurrence rate was reported as 40-60\% in those patients who had previously suffered from ICP [20]. Although its etiology is not exactly known, it presumably develops in genetically predisposed individuals under the impact of environmental and pregnancy-related hormonal factors $[5,8,21]$. In several studies performed, genetic variations have been demonstrated in the FXR nuclear receptor, which is expressed in the liver, bowels, and gallbladder tissues; it functions in the homeostasis of bile acid metabolism and some bile acid transport proteins [19, 22, 23].

As a member of the FGF family, FGF-19 is a hormone that is expressed essentially in the ileum, but not in the liver under normal conditions [24, 25]. It functions in interorgan signaling between the bowels and liver, and it regulates the bile acids, glucose, lipids, and energy metabolism [25]. The bile acids secreted into the small bowel activate the FXR receptors in the enterocytes, leading to an increase in FGF-19 expression [10]. Then, FGF-19 enters the portal circulation and selectively binds to the FGFR4/ $\beta$-klotho receptors on the hepatocytes. The $\beta$-klotho transmembrane protein is required for the binding of FGF-19 to FGFR4 and the subsequent activation of the intracellular signaling mechanism [15]. Although FGFR4 is prevalent in many tissues, the tissue-specific effects of FGF-19 manifest themselves if $\beta$-klotho and FGFR4 are expressed concurrently. However, the expression of higher levels of these two biomarkers is confined to the liver [12, 15]. Activated FGFR4 decreases the CYP7A1 gene expression and suppresses bile acid synthesis [10, 26]. Moreover, it has been reported that the regulation of CYP7A1 is achieved not only through the FXR pathway, but also via a direct effect [17].
In studies performed in mice, it has been demonstrated that deficiencies in the FGF-15 (the mouse orthologue of human FGF-19), $\beta$-klotho, and FGFR4 signaling system lead to a decrease in the negative feedback mechanism, with a resultant increase in CYP7A1 expression and hypersecretion of bile acids [12, 13, 17, 27]. Therefore, functional impairment involving the FGF-19, $\beta$-klotho, and FGFR4 signaling system and lower serum levels of these biomarkers could be expected in patients with ICP. In a study performed in mutant mice, $\beta$-klotho deficiency caused an increase in the CYP7A1 expression and the amount of bile acids. In response to an increase in the amount of bile acids, the appropriate upregulation of intestinal FGF-15, which is the mouse orthologue of FGF-19, has been demonstrated [17]. However, in a large cohort of ICP cases, Dixon et al. investigated the candidate genetic loci for ICP susceptibility, but they did not identify a genetic variation in the FGF-19 locus [28]. In view of the lower serum levels of $\beta$-klotho in our study, particularly in the presence of previous ICP episodes, it can be argued that a deficiency in $\beta$-klotho expression may play a role in the pathophysiology of ICP. In some patients, even in the presence of normal levels of FGF-19, which requires $\beta$-klotho for physiological activity, functional impairment involving the FGF-19, $\beta$-klotho, and FGFR4 signaling system may predispose them to or contribute to the development of ICP under the impact of pregnancy-related hormones.

Incidentally, the negative feedback regulation of bile acid synthesis via the FGF-19, $\beta$-klotho, and FGFR4 signaling system constitutes an adaptive response to protect the liver from the hepatotoxic effects of bile acids [24, 26, 29, 30]. In a group of patients with extrahepatic cholestasis caused by pancreatic tumors, Schaap et al. demonstrated that the plasma FGF-19 levels and gene expression of FGF-19 in the liver were significantly higher in cholestatic patients than in control patients and patients who received a biliary stent, whereas the hepatic gene expression of the $\beta$-klotho cofactor was significantly lower [24]. Wunsch et al. demonstrated that the liver expression and serum protein levels of FGF-19 were significantly induced in patients with primary biliary cirrhosis (PBC) with prolonged cholestasis, which also correlates with the severity of the liver disease. Therefore, they speculated that FGF-19 could be an important sign of cholestatic liver injury [29]. Similarly, Li et al. observed that an increase in the circulating FGF-19 was correlated with the severity of cholestasis in patients with PBC. They concluded that the regulation of BA synthesis in response to cholestasis is controlled mainly by FGF-19 [30].

In light of these findings, higher FGF-19 levels may be anticipated as a response to increased 
bile acids in ICP. However, the similar levels in the two groups in our study may reflect the fact that the enterohepatic feedback mechanism, via the FGF-19, $\beta$-klotho, and FGFR4 signaling system, does not play a primary role in the pathophysiology of ICP, and that different regulatory mechanisms might be activated. Another possible explanation is that despite the increased bile acid and transaminase levels in the ICP group, the response in the liver injury protective mechanism is not remarkable. This is due to the transient and milder nature of ICP when compared to chronic cholestatic liver diseases in which the hepatocytes show enhanced FGF-19 expression and become an important source of FGF-19 to protect the liver from the hepatotoxic effects of bile acids [24, 29, 30]. Lastly, this condition may also reflect the inadequate secretion of the molecule or an abnormality in the negative feedback mechanism in some patients.

The low number of patients and the cross-sectional design may be considered limitations of this study. Since the primary design of the study was not intended to investigate recurrent cases of cholestasis, the low number of patients with ICP histories included in our study was another limitation.

In conclusion, although the FGF-19 and $\beta$-klotho levels did not differ from those observed in the control group, at least in some patients, one may suggest their roles in the pathophysiology of ICP. We believe that larger scale, prospective, clinical, and genetic studies that encompass the family members with histories of recurrent ICP may be beneficial in the elucidation of the pathophysiology of ICP involving the FGF-19, $\beta$-klotho, and FGFR4 signaling system. These could lead to the subsequent prevention of maternal and fetal complications, as well as the development of new treatment alternatives.

\section{Acknowledgments}

The authors thank MD Gokhan Yildirim for his help with the manuscript and his valuable advice in the statistical analysis.

\section{Conflic of interest}

The authors declare no conflict of interest.

\section{References}

1. Fagan EA. Intrahepatic cholestasis of pregnancy. Clin Liver Dis 1999; 3: 603-32.

2. Joshi D, James A, Quaglia A, Westbrook RH, Heneghan MA. Liver disease in pregnancy. Lancet 2010; 375: 594-605.

3. Ropponen A, Sund R, Riikonen S, Ylikorkala O, Aittomaki K. Intrahepatic cholestasis of pregnancy as an indicator of liver and biliary diseases: a population-based study. Hepatology 2006; 43: 723-8.
4. Geenes V, Chappell LC, Seed PT, Steer PJ, Knight M, Williamson C. Association of severe intrahepatic cholestasis of pregnancy with adverse pregnancy outcomes: a prospective population-based case-control study. Hepatology 2014; 59: 1482-91.

5. Tayyar AT, Tayyar A, Atakul T, et al. Could first- and second-trimester biochemical markers for Down syndrome have a role in predicting intrahepatic cholestasis of pregnancy? Arch Med Sci 2017; doi: 10.5114/ aoms.2017.69865.

6. Gutaj P, Wender-Ożegowska E, Brązert J. Maternal lipids associated with large-for-gestational-age birth weight in women with type 1 diabetes: results from a prospective single-center study. Arch Med Sci 2016; 13: 753-9.

7. Kulhan M, Kulhan NG, Nayki U, Nayki C, Ata N. Intrahepatic cholestasis of pregnancy and fetal outcomes. Mini review. Arch Med Sci Civil Dis 2017; 2: 85-6.

8. Arrese M, Macias RI, Briz O, Perez MJ, Marin JJ. Molecular pathogenesis of intrahepatic cholestasis of pregnancy. Expert Rev Mol Med 2008; 10: e9.

9. Koroglu N, Tayyar A, Tola EN, et al. Increased levels of the novel hepatokine Fetuin B in patients with intrahepatic cholestasis of pregnancy. J Matern Fetal Neonatal Med 2017; doi: 10.1080/14767058.2017.1413546.

10. Beuers U, Trauner M, Jansen P, Poupon R. New paradigms in the treatment of hepatic cholestasis: from UDCA to FXR, PXR and beyond. J Hepatol 2015; 62: S25-37.

11. Holt JA, Luo G, Billin AN, et al. Definition of a novel growth factor-dependent signal cascade for the suppression of bile acid biosynthesis. Genes Dev 2003; 17: 1581-91.

12. Kuro-o M. Endocrine FGFs and Klothos: emerging concepts. Trends Endocrinol Metab 2008; 19: 239-45.

13. Inagaki T, Choi M, Moschetta A, et al. Fibroblast growth factor 15 functions as an enterohepatic signal to regulate bile acid homeostasis. Cell Metab 2005; 2: 217-25.

14. Wu X, Ge H, Gupte J, et al. Co-receptor requirements for fibroblast growth factor-19 signaling. J Biol Chem 2007; 282: 29069-72.

15. Lin BC, Wang M, Blackmore C, Desnoyers LR. Liver-specific activities of FGF19 require Klotho beta. J Biol Chem 2007; 282: 27277-84.

16. Ornitz DM, Itoh $\mathrm{N}$. The fibroblast growth factor signaling pathway. Wiley Interdiscip Rev Dev Biol 2015; 4: 215-66.

17. Ito S, Fujimori T, Furuya A, Satoh J, Nabeshima $Y$, Nabeshima Y. Impaired negative feedback suppression of bile acid synthesis in mice lacking betaKlotho. J Clin Invest 2005; 115: 2202-8.

18. Ahmed KT, Almashhrawi AA, Rahman RN, Hammoud GM, Ibdah JA. Liver diseases in pregnancy: diseases unique to pregnancy. World I Gastroenterol 2013; 19: 7639-46.

19. Williamson C, Geenes V. Intrahepatic cholestasis of pregnancy. Obstet Gynecol 2014; 124: 120-33.

20. Hepburn IS, Schade RR. Pregnancy-associated liver disorders. Dig Dis Sci 2008; 53: 2334-58.

21. Tayyar A, Temel Yuksel I, Koroglu N, et al. Maternal copeptin levels in intrahepatic cholestasis of pregnancy. J Matern Fetal Neonatal Med 2017. doi: 10.1080/ 14767058.2017.1335708.

22. Van Mil SW, Milona A, Dixon PH, et al. Functional variants of the central bile acid sensor FXR identified in intrahepatic cholestasis of pregnancy. Gastroenterology 2007; 133: 507-16.

23. Bacq Y, Sapey T, Brechot MC, Pierre F, Fignon A, Dubois F. Intrahepatic cholestasis of pregnancy: a French prospective study. Hepatology 1997; 26: 358-64.

24. Schaap FG, van der Gaag NA, Gouma DJ, Jansen PL. High expression of the bile salt-homeostatic hormone fibro- 
blast growth factor 19 in the liver of patients with extrahepatic cholestasis. Hepatology 2009; 49: 1228-35.

25. Angelin B, Larsson TE, Rudling M. Circulating fibroblast growth factors as metabolic regulators: a critical appraisal. Cell Metab 2012; 16: 693-705.

26. Kliewer SA, Mangelsdorf DJ. Bile acids as hormones: the FXR-FGF15/19 pathway. Dig Dis 2015; 33: 327-31.

27. Yu C, Wang F, Kan M, et al. Elevated cholesterol metabolism and bile acid synthesis in mice lacking membrane tyrosine kinase receptor FGFR4. J Biol Chem 2000; 275: 15482-9.

28. Dixon PH, Wadsworth CA, Chambers J, et al. A comprehensive analysis of common genetic variation around six candidate loci for intrahepatic cholestasis of pregnancy. Am J Gastroenterol 2014; 109: 76-84.

29. Wunsch E, Milkiewicz M, Wasik U, et al. Expression of hepatic fibroblast growth factor 19 is enhanced in primary biliary cirrhosis and correlates with severity of the disease. Sci Rep 2015; 5: 13462.

30. Li Z, Lin B, Lin G, et al. Circulating FGF19 closely correlates with bile acid synthesis and cholestasis in patients with primary biliary cirrhosis. PLoS One 2017; 12 e0178580. 